\title{
Towards Game-Guided Exploration Systems for Self-Facilitated Exhibitions
}

\author{
Rameshnath Krishnasamy \\ Aalborg University \\ Teglgaards Plads 1, $11^{\text {th }}$ fl., rm. 11.01 \\ Denmark \\ krishnasamy@id.aau.dk
}

\begin{abstract}
Automated exhibition sites require facilitation and mediation beyond signage and text labels to guide and sustain user engagement throughout the visit. Museums and cultural heritage sites have a rich history of experimenting with games and playful experiences to enable curiosity and motivate users to explore and engage with the exhibition content, yet the challenge of selffacilitation in automated sites brings unexplored areas into both existing research and practices. Rapid technological advancements have further developed the digital frontier, placing context aware, mixed reality applications and game systems in the hands of a growing number of users. This offers a renewed interest in investigating facilitation in automated sites mediated through games that utilise emerging technologies. However, this optimistic view is tempered by our limited understanding of how to design exhibition games to support the user in self-facilitated situations. This paper introduces the Game-Guided Exploration Systems framework as a way to think and talk about games and playful experiences for facilitation and mediation in automated exhibitions. The framework is based on gaming schemas extracted from game literature (rules, play and context) supported by perspectives on facilitation and mediation. The paper illustrates this framework, which is derived from investigating existing game systems, theory and through the development of a game system designed to support self-facilitation through play and exploration.
\end{abstract}

Design. Human factors. Museum. Human-Computer Interaction. Playful interaction. Gamification.

\section{INTRODUCTION}

Recently, a trend in exhibition sites towards automation and self-facilitation of the user experience places digital technologies at the centre of mediation and communication strategies, fostering playful interactions between the user, the physical place and digital space. These types of digital context aware experience layers have been pertinent to human-computer interaction $(\mathrm{HCl})$ research that revolves around museums and cultural heritage sites, established as a subfield and denoted as human-exhibition interaction (HEI) (Wang \& Xia 2019). Existing research shows technology-centred investigations, where emerging technologies take centre stage and the museological context is a playground (Wang \& Xia 2019), while other research points to specific challenges, such as facilitation, mediation and communication strategies. The work presented here is part of a Ph.D. research project which in turn is one out of thirteen other Ph.D. projects, under a national research initiative in Denmark entitled "Our Museum" (Our Museum 2016) that aims to investigate perspectives on past, present and future museum communication and mediation practices. The research project that this paper is part of aims to investigate design strategies for digital communication, facilitation and mediation in automated exhibition sites.

Based on the current understanding of exhibition games and the use of games, gamification and playful systems to enrich the user experience in exhibitions (Flanagan 2009; Witcomb 2013), the following hypotheses is produced:

Games can be designed to enable exploratory behaviour, through emotional triggers, such as curiosity, which in turn leads to discovery and ultimately knowledge acquisition.

A requirement for this is, that the game does not instruct the user to do anything forcibly, but merely suggest, so the decision to explore, the level of information absorption, and selection of exhibits, lies with the user. The system should enable exploratory behaviour, provide guidance and 
incentivise discovery. In other words, the game system provides the extrinsic motivation designed to support the users own intrinsic motivations. From there, it is up to the individual user to decide what they want to explore and how much knowledge they wish to absorb. This approach is absent in the design of game systems and gamification of exhibition sites. Most existing game systems or attempts at bringing play mediated interactions, are directed towards specific learning goals even though the broad agreed upon understanding of the exhibition context is that it is an informal learning space with a variety of user needs and motivations (Falk \& Dierking 2000; Simon 2010). This research project aims to investigate and contribute to expanding the field of game guided exploration systems.

\section{BACKGROUND}

In recent times, many sites and landmarks have come under 'severe long-term challenges', due to economic and political reasons (Lindqvist 2012) citing changes in experience economical tendencies (Skot-Hansen 2008). In response to these challenges, centralization strategies and efficiency measures have been initiated, which implies a decommissioning of smaller or challenged exhibition sites. Despite being international icons for tourism and the socio-economic impact cultural heritage sites and museums are capable of contributing with (N 2017; Piekkola, Suojanen \& Vainio 2014) This in turn has encouraged institutions to turn their gaze towards automation as a possibility to preserve sites and keep the exhibitions operational (Krishnasamy 2016).

A recent user survey in Denmark showed a number of key factors contributing to the absence and decline of users. In this survey, two types of users were identified: users and non-users. Users are frequent visitors and consist primarily women and aging audiences; and non-users are everyone else, who rarely, if ever, visit. Non-users are cited to perceive the exhibitions as 'old and dusty' and 'boring', pointing to a lacklustre experience compared to other attractions. A strategy inspired by an 'outcome-driven innovation' approach (Ulwick 2002) has inspired institutions to investigate what other cultural offers the non-users are seeking and prioritizing over museums. The survey provides insight about this and reveals that the two top competitors that attract the potential users are cinema and music events. This discourse has been debated since the early 90's as 'Disneyfication' (McLean 1993; Terrell 1991) with examples dating back to 1970's. However, looking at the survey from 2018, this paradigm is still relevant. Users have come to expect blockbuster experiences and are quickly demotivated or discouraged from visiting an exhibition instead. Furthermore, removing all personnel and replacing all aspects of communication with signage, posters, labels and encased artefacts in display cages limits the potential of mediation. Exhibitions relying on these types of communication and mediation strategies struggle to sustain and/or attract (non)users. This is evident in the numerous research and development initiatives funded by both private and public entities in the EU, such as EuNaMus, MeLa, Europeana and the meSch projects; and more locally in Denmark, such as the Our Museum and GIFT projects from more recent times. These all have in common to develop and sustain exhibitions, and a large number of these projects stem from $\mathrm{HCl}$ research with attention to how emerging and existing digital technologies can intervene and take on challenges. This has led institutions to a focused aim at integrating digital mediation into exhibitions to support users in self-facilitated situations.

Relying on digital experience layers integrated in the exhibition, such as displays (Claisse, Petrelli, Dulake, Marshall \& Ciolfi 2018), augmented and virtual reality (Jung, tom Dieck, Lee \& Chung 2016), audio augmentations(Antoniou, O'Brien, Bardon, Barnes \& Virk 2015), amongst a host of other digital mediation strategies and technologies, to enrich the user experience through interaction, have been covered extensively in the literature, with mixed results such as (Kuflik 2012; Kuflik, Wecker, Lanir \& Stock 2015; Lykke \& Jantzen 2016). This approach was however not pursued for the research project this study is part of, partly because it has an existing body of research, and partly due to the nature of remote, automated exhibition sites that rely heavily on self-facilitation. Previous research has showed the detrimental user experience of having to wait for other users to finish a session or blocking the view to either artefact or labels. Sometimes referred to as hyper congestion (Khan, Krishnasamy \& Germak 2018) and could be a contributing factor to the fatigue found in exhibitions (Bitgood 2009). Any interventions on exhibition sites needs sensitive design, careful integration and planning (Claisse et al. 2018). The most prevalent platform to rely on was the pervasive and ubiquitous smartphone. In doing so this project adopts the BYOD ("bring your own device") approach, which has been a way to work around the economical and practical challenges with automated sites where it is simply not possible to rent or offer devices to the users. Using smartphones does not affect the existing heritage landmarks or artefacts, it is a way to integrate digital technologies without causing any damage or modifying the historical objects, instead the technology provides ubiquitous platform to augment and integrate the exhibition into a game system. 
Today, smartphones provide a growing collection of tools for our working lives, social lives, and personal entertainment (Harper 2008). They extend and support personal, cognitive and social processes such as habit-changing, problemsolving, creating, analysing, learning or performing a skill (Rogers 2006) and spanning areas such as healthcare, education, entertainment, tourism, banking and governance. The influence of smartphones is evident in the explosion of devices over the past decade ("Ericsson Mobility Report MWC," 2019). Rapid technological advancement combined with smartphones becoming universally owned has given rise to an abundance of emerging applications that exploit the possibilities of these devices. The focus here is on applications that bridge the user with digital spaces and physical places, which may be appropriated to selffacilitated exhibitions.

Currently games provide the most resilient genre for establishing that link between the user and the world around them, found most suitable for designing a system to support the user in selffacilitation exhibition sites, that also includes digital experiences to attract and engage users as an alternative to signs, labels and posters.

One of the most influential examples from recent times is Pokémon GO, which changed the way games can be played for most users. There exist many location- and context aware games for smartphones, but none came close to the number of new and sustained users of Pokémon GO. This and similar games have demonstrated their capacity to attract and introduce typical nongamers to a novel way of playing in addition to drawing traditional gamers out of their habitats and into the world.

Although they are not marketed as fitness or exercise games, they require exertion and physical activity to be played, and game mechanics are employed to enable social interaction, such as cooperative or competitive play modes. The novelty effect of such games is powerful, and if designed properly, they can sustain user engagement over long periods of time.

Commercial products, such as Pokémon GO, along with a number of research projects (R. A. Ballagas et al. 2007; Chang, Hou, Pan, Sung \& Chang 2015; Wakkary et al. 2009) from the HEl field and systems designed from this project (Khan et al. 2018; Krishnasamy 2018, 2018) have all contributed to a design space that highlights the value of similar mediated exhibition experiences.

This will henceforth be referred to as exhibition games, although the author realises that this is a term used in a sports context to describe a certain type of sport event. Here exhibition games are defined as a digital game that is played in an exhibition site where the user is linked to the content and context of the site through playful interaction.

Research into human-exhibition interaction is important, as exhibitions are capable of positive socio-economic impact which includes mental health benefits (Silverman 2010), support socialization (Sacco \& Crociata 2013). But, if there is a decline in attendance or users opting for other attractions, the future of exhibition sites is at risk.

The research presented here aims to tackle the twin challenge of designing digital games that support self-facilitation at automated exhibitions without tampering with or interfering with the site or the artefacts and objects displayed here and to provide an alternative experience than what the non-users have come to expect, drawing from games to design systems that can guide the users through playful interaction. When designing for exhibition games, the user's role in an exhibition must be understood, as well as the technological challenges of supporting the user in self-facilitated situations. Additionally, when discussing facilitation in exhibitions, there is an important distinction to be made; there are, here, two dimensions of facilitation. One can be translated to 'guidance' and denotes wayfinding and information retrieval while moving physically within the exhibition site while the other dimension refers to facilitation from a learning perspective. These two dimensions will not be further elaborated in this paper. However, it is important to underline that the point of departure for this research treats facilitation as both dimensions combined, and therefore designing games for this context should include ways to address both dimensions.

There are general game design frameworks (Hunicke, LeBlanc \& Zubek 2004; Tekinbaş \& Zimmerman 2003), and many that discuss how to implement games in exhibitions and the implications and potentials thereof (Beale 2011). However, there is at present a limited understanding of how to design and develop exhibition games that can support the user in selffacilitated exhibitions.

Therefore, the goal here is to present design strategies gathered through existing exhibition games with a framework to encourage a systematic consideration of how to design game guided systems for exhibitions. This includes discussing how game design and which technologies can support such experiences. Technologies are naturally a critical aspect, as all research done before, also often explores the status quo of new technology's potential to mediate. Here, emerging 
technologies of today will also be considered in addressing the challenges.

Thus, the research question that guides this paper is, how can emerging technologies and gamification be integrated into a framework to support the user in self-facilitated exhibitions? The work will be based on ethnographic studies of exhibitions, review of existing systems, a look into the literature and finally through studies from game systems developed by the author to address aspects of the presented design challenge. However, most of the data will only briefly be touched in this paper as they are presented elsewhere where the methodological approach is discussed in depth.

The resulting framework, the Game-Guided Exploration System will be presented as a conversation tool, which discusses design dimensions and through these propose design tactics for other designers, developers and practitioners and researchers to make use of. It also shapes the baseline for more work and future related studies of this research project. It does so by analysing a mixed reality game that was developed as part of the research project.

\subsection{HALs museum}

The Our Museum research initiative is, as mentioned in the introduction a constellation of museum institutions and universities. The institutions have dual roles in this project; they provided a specific challenge they are facing, and they provide an exhibition to be used as a research site. The Historical Museum of Northern Jutland (HMNJ), the collaborating institution in this project, pointed to the challenge of transitioning to fully automated, self-facilitated sites and provided an old redoubt with the arsenal building. This exhibition site is now part house museum (inside the old arsenal building), part exhibition site. See Figure 1 below.

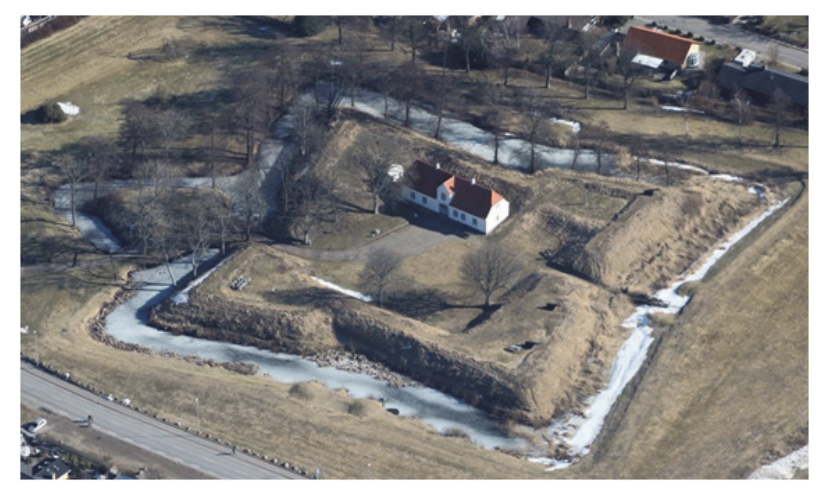

Figure 1: Aerial shot of the redoubt and the remaining house, which now serves as part house museum, part traditional exhibit.
It is located in a small Danish village in Northern Jutland, called Hals. This is the first exhibition site in line to transition over to fully automated operation, and therefore offered as site for conducting tests and studies. The collaborating institution Originally, the plan was to end the research project and then re-design the house as a self-facilitated site, but due to external, political, forces, the house transitioned halfway through. The implication of the accelerated transition is that the re-design of the space, place and inventory happened in parallel without any link to the research project. The result was a game that was developed as a layer on top of the existing exhibition - a mixed reality game system. The

\subsection{Discover the redoubt}

The game was designed through co-design with museum professionals from the institution, to ensure that the content matched with the vision of the upcoming re-designed exhibition as well as the integrity and factual accuracy of the presented content. The author participated throughout the design project and acted as facilitator of co-design workshops, which included educators and museum personnel that had been at Hals Museum for over 15 years, curators, custodians and facilitators. The role of the author changed from $\mathrm{HCl}$ researcher, to designer, developer and game designer through the design process. The design process itself, was based on baseline studies of the exhibition site; user surveys, observation studies, interviews, shadowing to name a few methods used to gather data that would later inform the design of the game. This resulted in a concept handbook that was used as basis to develop a variety of game concepts across a number of technology types.

The game system design was influenced from past research projects such as REXPLORER, a game designed for handheld devices in outdoor exhibition settings that would encourage the users to move around and investigate hidden objects to reveal information (R. Ballagas, Kuntze \& Walz 2008); PHANTOM (Jensen, Krishnasamy \& Selvadurai 2010) a pervasive game developed for smartphones to motivate users to physically move around in order to complete game tasks in order to combat sedentary lifestyles and instigate physical activity; along with more recent commercial examples such as Pokémon GO.

The design was guided by game design theory backed by related work from gamification. In early stages, the Mechanics-Dynamics-Aesthetics framework (Hunicke et al. 2004) was used to analyse as well as identify key components such as how emotions (the aesthetics component) can be used as triggers to enable certain behaviours. This led to focusing on exploration as a key behaviour to 
encourage the user to roam the 'game map' and simultaneously opened up investigation into emotional triggers to instigate certain behaviours.

The result is a mixed reality game, DISCOVER THE REDOUBT (translated from Danish "Opdage Skansen"); a bespoke location-based scavenger hunt/quiz application for smartphones, that utilises Bluetooth beacons. The game is played on the redoubt, both indoors and outside utilising the whole area as a game map. There are three parts; 1: the area outside, 2: the powder chamber and 3: the arsenal, see Figure 2 below. The game itself has two game modes: one is a scavenger hunt/quiz in line with many other traditional exhibition games, and the other is based exclusively on unlocking content by exploring. The only instructions available are indicators that tell the user an approximate distance to a beacon.

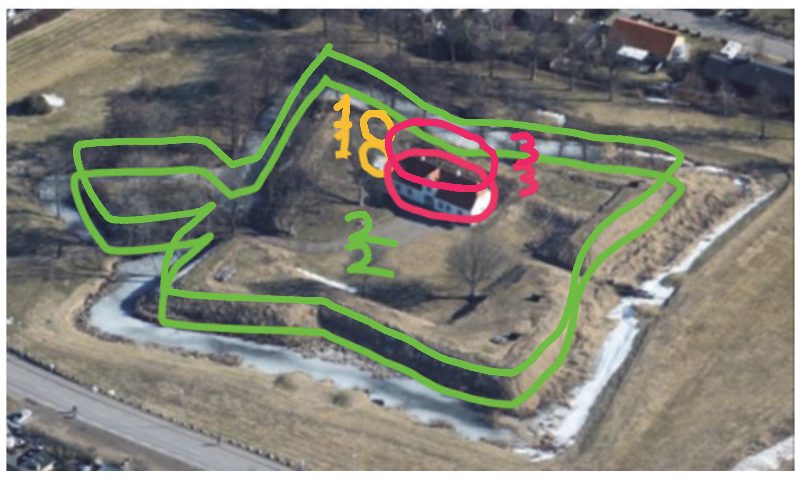

Figure 2: The game map within the mixed reality game, showing the two 'instances' (1+3) along with the 'overworld' (2), using game design vocabulary.

The primary goal of the game is to motivate users to explore the exhibition site. To achieve this, Bluetooth beacons were placed in strategic points of interest $(\mathrm{POI})$ that unlocks content within the game, when the user moves into close proximity of the beacon. The game layer presents the user with a view of the redoubt from the 1600's when it was built, and by unlocking POls the user would reveal the original architecture of the redoubt, see Figure 3.

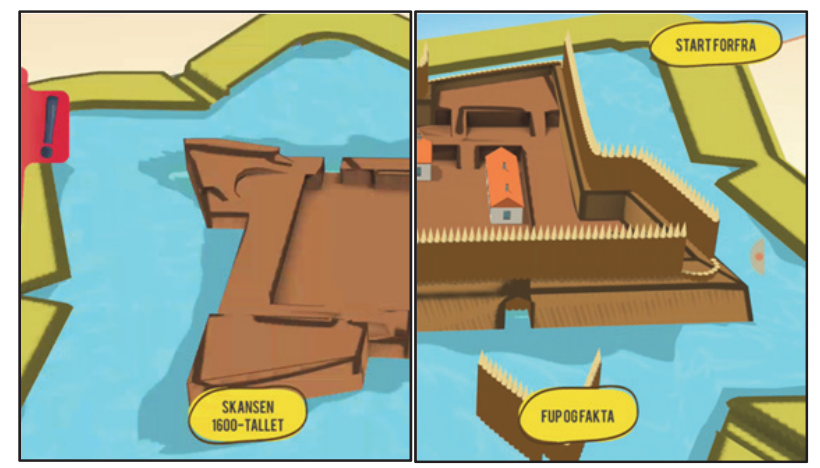

Figure 3: Left: "empty" layer the user sees in the Explore game mode. Right: fully unlocked map.
The quiz mode provides the user with a contemporary map of the exhibition, see Figure 4, with quizzes popping up through characters that are tied to that specific exhibit. For instance, a fisherman asks "true or false" questions about fishes.

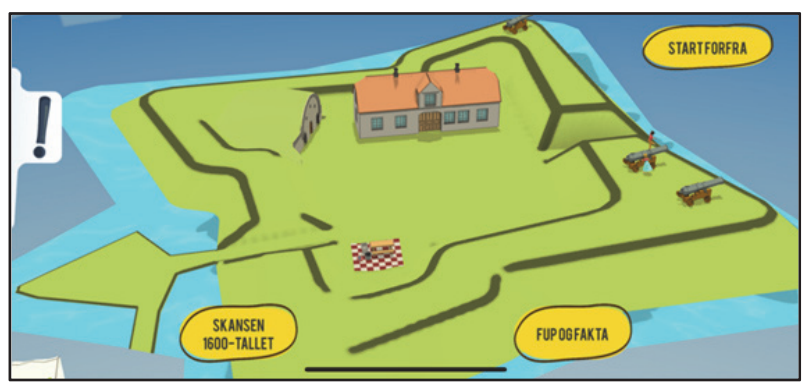

Figure 4: This presents the user with a contemporary representation of the exhibition site and contains quizzes that are related to the artefacts and objects displayed inside the house.

The game was tested twice; once as a lab test with a group of approximately 50 students, who then evaluated the game experience and subsequently a different group of 21 students in situ. A mix of Google analytics, user surveys, group discussions and observation studies were used to collect and capture qualitative and quantitative data.

In summary, the game system presented supports self-facilitation through exploration around, both inside and outside, the exhibition site through gaming experiences; it engages users through interaction with POls that bridges the digital space on the smartphone with the physical place and objects on-site; and it facilitates digitally mediated user engagement through play. Social play modes emerged without any direct input from the game system.

Findings revealed insight about how they played the game, in solitude and socially. See Figure 5.

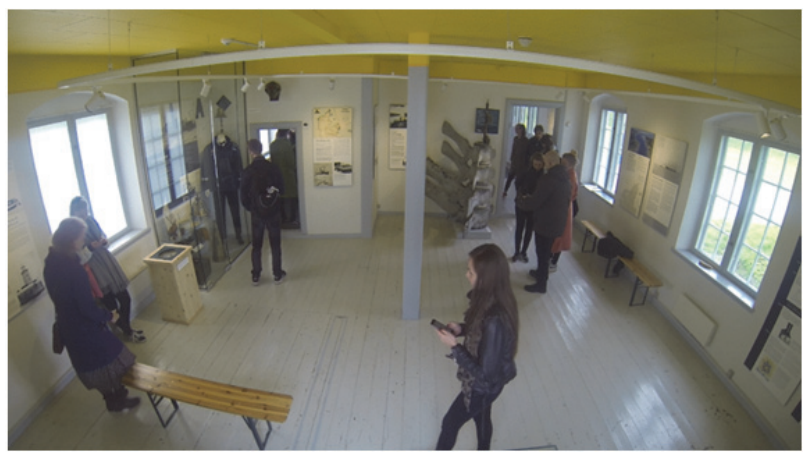

Figure 5: In situ user test revealed different play modes that emerged from the context; single player as well as cooperative and competitive multiplayer modes were observed. 
One interpretation could be that this relates back to type of user. This aspect has been covered in the existing literature as users in $\mathrm{HCl}$ studies relating to the user experience (Battarbee \& Koskinen 2005); visitor in $\mathrm{HEl}$ studies relating to the visitor experience (Bitgood \& Shettel 1996); and player in game studies relating to the player experience (Chu, Wong \& Khong 2011). This perspective is intriguing because existing literature covers humansystem interaction in respect to a specific field, but in designing exhibition games, it should provide a better vocabulary to work with if the three contexts were aligned.

\section{TOWARDS A GAME-GUIDED EXPLORATION SYSTEM}

Based on existing games research, humanexhibition interaction literature and commercial games, a mixed reality game was designed targeting the challenge of self-facilitation in automated sites. The aim was to provide the users with a game that can support their visiting experience and facilitate both guidance as well as learning about the exhibition in an indirect playful way.

A design strategy was formulated and through that, a preliminary framework was established to guide the design of "Discover the Redoubt". Initially openworld, adventure and exploration themed games, such as Breath of the Wild, Minecraft, Journey and Pokémon GO were analysed using learning and game theories and used as source for inspiration to design the game. Drawing inspiration from the framework for exertion games (Mueller et al. 2011), gaming schemas, rules, play and culture from games literature (Tekinbaş \& Zimmerman 2003) were appropriated and used to explore formal structures that could benefit in designing exhibition games.

\footnotetext{
"RULES contain formal game design schemas that focus on the essential logical and mathematical structures of a game. PLAY contains experiential, social, and representational game design schemas that foreground the player's participation with the game and with other players. CULTURE contains contextual game design schemas that investigate the larger cultural contexts within which games are designed and played." (Tekinbaş \& Zimmerman 2003)
}

By using the schemas, a vocabulary started to emerge that describes parts of the game. The schema play: play as pleasure, where rule-bound and autotelic play supports extrinsic and intrinsic motivation. The schema rules: emergent systems support exploration in a nonlinear way, but points to all directions and leaves the choice and sequence up to the user. The third schema culture considers the immediate context as well as the broader perspective; it is being played in an exhibition site, but it is also part of an institution that serves as a catalyst for socio-economic impact. These were used as design principles informing the concept development.

However, after the initial user tests, it became clear that there is space for improvement regarding user characteristics. The emergent play style adopted showed potential to extend the game to better accommodate different types of users in exhibitions. As a result of the findings from the user test, a more structured approach to create an overview and align the three aspects has been attempted in the arrangement below. It uses the three schemas framework to inform the design dimension relating to the user. On the opposite end of each schema is a corresponding part of the challenge the research set out to investigate; facilitation, mediation and context. See table 1 below.

Table 1: A preliminary arrangement of the elements of exhibition games of the exhibition games framework.

\begin{tabular}{|c|c|c|c|c|}
\hline & USER & VISITOR & PLAYER & \\
\hline RULES & GUIDANCE & LEARNING & EXPLORATION & FACILITATION \\
\hline PLAY & TECCHNOLOGY & INFORMATION & GAME & MEDIATIIN \\
\hline CULTURE & ENVIRONMENT & EXHIBITION & MAP/LEVEL & CONTEXT \\
\hline
\end{tabular}

Each of the dimensions require a more elaborate explanation, but the overall idea is to use this to discern between what constitutes a system for the user to interact with, what constitutes an exhibition the visitor visits with and what constitutes a game that the player plays. These have then been arranged according to the design challenge they address, for instance self-facilitation is addressed through the RULES schema, where guidance is user related, and learning is visitor related and exploration is player related. But through design tactics that combines all the dimensions offer new ways to support the user in self-facilitated exhibitions.

\section{DISCUSSION AND CONCLUSIONS}

In this paper a preliminary framework has been presented, based on findings from user tests with a mixed reality game designed to support users at automated exhibitions. It has shown how to support novel exhibition game concepts, but further work is needed to substantiate how the framework can be operationalised to design and implement game systems. It does however provide a staging ground for a design vocabulary that can be further developed and iterated upon.

From the user tests, one thing that stood out was the way these systems should be designed to embrace as many different types of users as possible. It is clear that looking at what motivates 
human beings was a better point of origin for designing exhibition game systems. This has led to drawing a parallel between experience and the overarching human-system interaction, with three underlying roles; a user of a system; a visitor in an exhibition; and a player in a game. Further research will pursue a clarification and alignment of these three aspects in order to provide a better understanding of how to design exhibition games.

\section{ACKNOWLEDGEMENTS}

The author wants to acknowledge the work by Professor Florian Mueller and his crew in "Designing Sports: A Framework for Exertion Games" (Mueller et al. 2011) that inspired the approach this paper.

\section{REFERENCES}

Antoniou, A., O'Brien, J., Bardon, T., Barnes, A. and Virk, D. (2015) Micro-augmentations: situated calibration of a novel non-tactile, peripheral museum technology (pp.229-234). ACM Press. https://doi.org/10.1145/2801948.2801959

Ballagas, R. A., Kratz, S. G., Borchers, J., Yu, E., Walz, S. P., Fuhr, C. O., ... Tann, M. (2007) REXplorer: A Mobile, Pervasive Spell-casting Game for Tourists. In $\mathrm{CHI}$ '07 Extended Abstracts on Human Factors in Computing Systems (pp.1929-1934). New York, NY, USA: ACM. https://doi.org/10.1145/1240866.1240927

Ballagas, R., Kuntze, A. and Walz, S. P. (2008) Gaming Tourism: Lessons from Evaluating REXplorer, a Pervasive Game for Tourists. In J. Indulska, D. J. Patterson, T. Rodden and M. Ott (eds), Pervasive Computing (Vol. 5013, pp.244261). Berlin, Heidelberg: Springer Berlin Heidelberg. https://doi.org/10.1007/978-3-54079576-6 15

Battarbee, K. and Koskinen, I. (2005) Coexperience: user experience as interaction. CoDesign, 1(1), 5-18.

https://doi.org/10.1080/15710880412331289917

Beale, K. (ed.). (2011) Museums at Play: Games, Interaction and Learning. Edinburgh: MuseumsEtc.

Bitgood, S. (2009) Museum Fatigue: A Critical Review. Visitor Studies, 12(2), 93-111. https://doi.org/10.1080/10645570903203406

Bitgood, S. and Shettel, H. H. (1996) An Overview of Visitor Studies. Journal of Museum Education, 21(3), 6-10.

https://doi.org/10.1080/10598650.1996.11510329
Chang, Y.-L., Hou, H.-T., Pan, C.-Y., Sung, Y.-T. and Chang, K.-E. (2015) Apply an Augmented Reality in a Mobile Guidance to Increase Sense of Place for Heritage Places. Educational Technology \& Society, 18(2), 166-178.

Chu, K., Wong, C. Y. and Khong, C. W. (2011) Methodologies for Evaluating Player Experience in Game Play. In $\mathrm{HCl}$ International 2011 - Posters' Extended Abstracts (pp.118-122). Springer, Berlin, Heidelberg. https://doi.org/10.1007/978-3-64222098-2 24

Claisse, C., Petrelli, D., Dulake, N., Marshall, M. and Ciolfi, L. (2018) Multisensory interactive storytelling to augment the visit of a historical house museum. In Proceedings of the 2018 Digital Heritage International Congress. IEEE. http://shura.shu.ac.uk/22646/ (retrieved 18 March 2019).

Ericsson Mobility Report - MWC. (2019), 28.

Falk, J. H. and Dierking, L. D. (2000) Learning from museums: visitor experiences and the making of meaning. Walnut Creek, CA: AltaMira Press.

Flanagan, M. (2009) Critical play: radical game design. Cambridge, Mass. London, England: MIT Press.

Harper, R. (ed.). (2008) Being human: humancomputer interaction in the year 2020. Cambridge, England: Microsoft Research.

Hunicke, R., LeBlanc, M. and Zubek, R. (2004) MDA: A formal approach to game design and game research. In Proceedings of the AAAI Workshop on Challenges in Game Al (Vol. 4).

http://www.aaai.org/Papers/Workshops/2004/WS04-04/WS04-04-001.pdf (retrieved 18 March 2019).

Jensen, K. L., Krishnasamy, R. K. and Selvadurai, V. (2010) Studying PH.A.N.T.O.M. in the Wild: A Pervasive Persuasive Game for Daily Physical Activity. Presented at the 22nd Conference of the Computer-Human Interaction Special Interest Group of Australia on Computer-Human Interaction, Association for Computing Machinery. https://doi.org/10.1145/1952222.1952228

Jung, T., Tom Dieck, M. C., Lee, H. and Chung, N. (2016) Effects of Virtual Reality and Augmented Reality on Visitor Experiences in Museum. In A. Inversini \& R. Schegg (eds), Information and Communication Technologies in Tourism 2016 (pp.621-635). Cham: Springer International Publishing. https://doi.org/10.1007/978-3-31928231-2 45 
Khan, S., Krishnasamy, R. and Germak, C. (2018) Design challenges in promoting inclusion for cultural heritage contents through low cost technology. DS 91: Proceedings of NordDesign 2018, Linköping, Sweden, 14th - 17th August 2018.

https://www.designsociety.org/publication/40914/D esign+challenges+in+promoting+inclusion+for+cult ural+heritage+contents+through+low+cost+technol ogy (retrieved 18 March 2019).

Krishnasamy, R. (2016) Project 07: Self-facilitation in Automated Exhibition Sites (Research Proposal).

Aalborg University.

http://ourmuseum.dk/projects/programmeprojects/unmanned-exhibition-sites/ (retrieved 18 March 2019).

Krishnasamy, R. (2018) Integrating Smart Objects into Self- Guided Exhibitions: Challenges of Supporting Self-Guided Exhibitions through Nonidiomatic Technologies, 6.

Kuflik, T. (2012) Ubiquitous Display Environments: An Overview. In Ubiquitous Display Environments (pp.1-6). Springer, Berlin, Heidelberg. https://doi.org/10.1007/978-3-642-27663-7 1

Kuflik, T., Wecker, A. J., Lanir, J. and Stock, O. (2015) An integrative framework for extending the boundaries of the museum visit experience: linking the pre, during and post visit phases. Information Technology \& Tourism, 15(1), 17-47. https://doi.org/10.1007/s40558-014-0018-4

Lindqvist, K. (2012) Museum finances: challenges beyond economic crises. Museum Management and Curatorship, 27(1), 1-15. https://doi.org/10.1080/09647775.2012.644693

Lykke, M. and Jantzen, C. (2016) User Experience Dimensions: A Systematic Approach to Experiential Qualities for Evaluating Information Interaction in Museums. In Proceedings of the 2016 ACM on Conference on Human Information Interaction and Retrieval (pp.81-90). ACM.

http://dl.acm.org/citation.cfm?id=2854965 (retrieved 18 March 2019).

McLean, K. (1993) Planning for People in Museum Exhibitions. Washington, DC: Assn of Science Technology Ctr.

Mueller, F. "Floyd," Edge, D., Vetere, F., Gibbs, M. R., Agamanolis, S., Bongers, B. and Sheridan, J. G. (2011) Designing sports: a framework for exertion games. In Proceedings of the 2011 annual conference on Human factors in computing systems - CHI '11 (p.2651). Vancouver, BC, Canada: ACM Press.

https://doi.org/10.1145/1978942.1979330

N, N. (2017) Museums as Economic Engines: A National Report (p.34).
Our Museum. (2016) Our Museum. Retrieved March 18, 2019. http://ourmuseum.dk/ (retrieved 18 March 2019).

Piekkola, H., Suojanen, O. and Vainio, A. (2014) Economic impact of museums. University of Vaasa Levon Institute.

Rogers, Y. (2006) Moving on from Weiser's Vision of Calm Computing: Engaging UbiComp Experiences. In P. Dourish \& A. Friday (eds), UbiComp 2006: Ubiquitous Computing (Vol. 4206, pp.404-421). Berlin, Heidelberg: Springer Berlin Heidelberg. https://doi.org/10.1007/11853565 24

Sacco, P. L. and Crociata, A. (2013) A Conceptual Regulatory Framework for the Design and Evaluation of Complex, Participative Cultural Planning Strategies: A strategic framework for cultural planning. International Journal of Urban and Regional Research, 37(5), 1688-1706. https://doi.org/10.1111/j.1468-2427.2012.01159.x

Silverman, L. H. (2010) The Social Work of Museums (1 edition). London; New York: Routledge.

Simon, N. (2010) The participatory museum. Santa Cruz, California: Museum 2.0.

Skot-Hansen, D. (2008) Museerne i den danske oplevelsesøkonomi. Samfundslitteratur.

Tekinbaş, K. S. and Zimmerman, E. (2003) Rules of play: game design fundamentals. Cambridge, Mass: MIT Press.

Terrell, J. (1991) Disneyland and the Future of Museum Anthropology. American Anthropologist, 93(1), 149-153.

Ulwick, A. W. (2002, January 1) Turn Customer Input into Innovation. Harvard Business Review, (January 2002). https://hbr.org/2002/01/turncustomer-input-into-innovation (retrieved 18 March 2019).

Wakkary, R., Hatala, M., Muise, K., Tanenbaum, K., Corness, G., Mohabbati, B. and Budd, J. (2009) Kurio: a museum guide for families. In Proceedings of the 3rd International Conference on Tangible and Embedded Interaction (pp.215-222). ACM. http://dl.acm.org/citation.cfm?id=1517712 (retrieved 18 March 2019).

Wang, N. and Xia, L. (2019) Human-exhibition interaction (HEI) in designing exhibitions: A systematic literature review. International Journal of Hospitality Management, 77, 292-302. https://doi.org/10.1016/j.ijhm.2018.07.009

Witcomb, A. (2013) Understanding the role of affect in producing a critical pedagogy for history museums. Museum Management and Curatorship, 28(3), 255-271.

https://doi.org/10.1080/09647775.2013.807998 